\title{
CONTRACTED MODULAR DIOPHANTINE INEQUALITIES AND NUMERICAL SEMIGROUPS
}

\author{
J. C. Rosales And J. M. URBAnO-BlanCO
}

Abstract. We study the set $\mathrm{T}(a, b, c)$ of all integer solutions to the Diophantine inequality $a x \bmod b \leqslant x-c$, with $a, b, c$ nonnegative integers and $b \neq 0$. We obtain an exact formula for the cardinality of $\mathbb{N} \backslash \mathrm{T}(a, b, c)$ and give an algorithm to decide whether or not a numerical semigroup can be represented as $\mathrm{T}(a, b, c) \cup\{0\}$.

Mathematics subject classification (2000): 20M14, $13 \mathrm{H} 10$.

Key words and phrases: Diophantine inequality, numerical semigroup, Frobenius number.

\section{REFERENCES}

[1] V. BARUCCI, D. E. DobBs AND M. Fontana, Maximality Properties in Numerical Semigroups and Applications to One-Dimensional Analytically Irreducible Local Domains, Memoirs of the Amer. Math. Soc., 598, (1997).

[2] J. L. RamíReZ Alfonsín, The Diophantine Frobenius problem, Oxford University Press, 2006.

[3] J. C. Rosales, Pseudo-symmetric modular Diophantine inequalities, Math. Inequal. Appl., 8, (4) (2005), 565-570.

[4] J. C. Rosales, Symmetric modular Diophantine inequalities, Proc. Amer. Math. Soc., 134, (2006), 3417-3421.

[5] J. C. RosALES, P. A. GARCÍA-SÁNCHEZ, Numerical semigroups having Toms' decomposition, to appear in the Canad. Math. Bull.

[6] J. C. Rosales, P. A. García-SÁncheZ and J. M. Urbano-Blanco, Modular Diophantine inequalities and numerical semigroups, Pacific J. Math., 218, (2005), 379-398.

[7] J. C. Rosales, P. A. García-SÁncheZ, J. I. García-García And J. M. URbano-Blanco, Proportionally modular Diophantine inequalities, J. Number Theory, 103, (2003), 281-294.

[8] A. Toms, Strongly perforated $K_{0}$-groups of simple $C^{*}$-algebras, Canad. Math. Bull., 46, (2003), $457-472$. 